\title{
The HLA-DRB1 Locus as a Genetic Component in Giant Cell Arteritis Mapping of a Disease-linked Sequence Motif to the Antigen Binding Site of the HLA-DR Molecule
}

\author{
Cornelia M. Weyand, Kevin C. Hicok, Gene G. Hunder, and Jörg J. Goronzy \\ Department of Internal Medicine, Division of Rheumatology, Mayo Clinic and Foundation, Rochester, Minnesota 55905
}

\begin{abstract}
Giant cell arteritis (GCA) is a granulomatous vasculitis affecting persons over 50 years of age. The inflammatory infiltrate, which is targeted at the aorta and its proximal branches, includes activated $\mathrm{CD4}^{+}$helper $\mathrm{T}$ cells, histiocytes, and giant cells. To investigate whether the genetic polymorphism of the HLA-DRB1 genes contributes to the local accumulation of activated $T$ cells, we have analyzed both HLA-DRB1 alleles in a cohort of 42 patients with biopsy-proven GCA. The majority of patients $(60 \%)$ expressed the $B 1 * 0401$ or $B 1 * 0404 / 8$ variant of the HLA-DR4 haplotype, both of which also represent the major genetic factors underlying the disease association in RA. GCA patients negative for the disease-linked HLA-DR4 alleles were characterized by a nonrandom distribution of HLA-DRB1 alleles. Sequence comparison among the allelic products identified in the GCA cohort demonstrated heterogeneity for the sequence polymorphism of the third hypervariable region (HVR), but homology for the polymorphic residues within the HVR2 of the HLA-DRB1 gene. The GCA patients shared a sequence motif spanning amino acid positions 28-31 of the HLA-DR $\beta 1$ chain. In the structural model for HLA-DR molecules, this sequence motif can be mapped to the antigen-binding site of the HLA complex, suggesting a crucial role of antigen selection and presentation in GCA. In contrast, the sequence polymorphism linked to RA has been mapped to the HVR3 of the HLA-DRB1 gene and translates into a distinct domain of the HLA-DR molecule, the $\alpha$-helical loop surrounding the antigen-binding groove. A consecutive case series study demonstrated that GCA and RA rarely co-occurred, supporting the interpretation that distinct functional domains of the HLA-DR molecule are implicated in the pathomechanisms of these two autoimmune diseases. (J. Clin. Invest. 1992. 90:2355-2361.) Key words: rheumatoid arthritis $\bullet$ vasculitis $\bullet$ antigen presentation - HLA complex
\end{abstract}

\section{Introduction}

Giant cell arteritis (GCA) ${ }^{1}$ is a clinicopathological syndrome characterized by a granulomatous vasculitis of the extracranial branches of the proximal aorta (1). In many populations GCA

Address correspondence to Cornelia Weyand, M.D., Mayo Clinic, 200 First Street, Southwest, Rochester, MN 55905. 1992.

Received for publication 6 April 1992 and in revised form 7 July

1. Abbreviations used in this paper: GCA, giant cell arthritis; HVR, hypervariable region; PMR, polymyalgia rheumatica.

The Journal of Clinical Investigation, Inc.

Volume 90, December 1992, 2355-2361 is the most common vasculitic syndrome in persons over $50 \mathrm{yr}$ of age and if not treated promptly may represent a life-threatening vascular disease $(2,3)$. The pathogenesis of the disease is essentially unknown. Clues to the understanding of the underlying pathomechanisms have come from the analysis of the cellular infiltrate damaging the wall of the involved vessels. A granulomatous inflammatory reaction with dense infiltrates of mononuclear leukocytes, usually including multinucleated giant cells, is typical for GCA (4). Immunohistochemical studies have demonstrated that $\mathrm{CD} 4^{+}$helper $\mathrm{T}$ lymphocytes represent the dominant cell population (5). Subsequent studies of these cells have shown that markers of $T$ cell activation are expressed locally, supporting the notion that a $\mathrm{T}$ helper celldriven immune response is the cause of the vascular damage. In general, $\mathrm{T}$ cells are activated when they specifically recognize a complex formed by a fragment of a foreign antigen and an autologous HLA molecule (6). No disease causative antigen has been identified in GCA. The characteristic histological findings of the inflammatory infiltrate accumulating around the internal elastic lamina have led to speculations that degenerated elastic fibers represent the autoantigen initiating and perpetuating the chronic immune response. The frequent acute onset of clinical symptoms accompanied by an intense acute phase reaction has also raised questions of an infectious agent playing at least a precipitating role.

In this report, we have studied allelic HLA polymorphisms that are important in determining immunoresponsiveness to exogenous antigens. An association of the disease with the HLA-DR4 haplotype has been described but reports on the distribution of HLA haplotypes in GCA patients have not been conclusive (7-11). To address the question whether gene products of the HLA class II complex might contribute to GCA, we have analyzed the functionally most important locus, the HLA-DRB1 locus, in a cohort of patients with biopsy-proven GCA. Three allelic variants of the HLA-DRB $1 * 04$ family were found to be overrepresented among GCA patients. Interestingly, GCA is linked to the very same allelic variants as RA. However, despite the overrepresentation of HLA-DRB 1 *04 in GCA and RA, the risk to develop both diseases is low, suggesting that distinct pathomechanisms underlie the immune abnormalities in both diseases. More importantly, we found a sequence motif spanning the amino acid positions $28-31$ that is shared by all GCA patients and that is different from that found in RA. This sequence stretch translates into a polymorphic site in the antigen-binding groove of the HLA-DR molecule, suggesting a crucial role in the selection and presentation of antigen to $\mathrm{T}$ lymphocytes.

\section{Methods}

Patients. 42 patients who were seen in the Division of Rheumatology, Mayo Clinic, between May 1990 and January 1992 were enrolled into 
the study. 22 patients were newly diagnosed, and 20 patients were diagnosed before 1991. All patients had characteristic histological findings on biopsy. 41 of the patients were Caucasian and 1 was Asian Indian. 32 patients were from a local geographic area. To assess the demographic and clinical characteristics of the study population, the files of all 42 patients were reviewed. Data for the following parameters were collected: age at onset of the disease; sex; presence of musculoskeletal pain confined to the shoulder and pelvic girdle, indicating symptoms of polymyalgia rheumatica (PMR); and permanent vision loss. To document permanent vision loss, atrophy of the optical nerve as diagnosed by an ophthalmologist was required. GCA patients diagnosed before 1989 were evaluated for relapses of the disease or requirement for longterm corticosteroid therapy. Patients were identified who required corticosteroid therapy for $>2 \mathrm{yr}$.

To identify patients who had biopsy-proven GCA as well as seropositive RA, we used a computerized search of the Mayo Clinic data base for the period 1976-1990. 835 patients carried the diagnosis of biopsyproven GCA. Within that cohort, 22 patients were diagnosed with polyarthritis. The charts of these 22 patients were reviewed to identify the underlying disease causing the polyarthritis. The diagnosis of polyarthritis secondary to GCA was verified in patients who were negative for rheumatoid factor and fulfilled the clinical and radiological characteristics described recently (12). RA and systemic lupus erythematosus were diagnosed according to the criteria of the American College of Rheumatology $(13,14)$. To estimate the expected numbers of cases of RA within a cohort of 835 patients, data of Linos et al. (15) were used. The prevalence of definite and classical RA in 72-yr-old individuals was calculated from the accumulated incidence rates for a cohort of 835 individuals.

Normal controls. A group of 63 individuals was analyzed for HLADRB1 alleles between 1990 and 1991. None of the normals had any current or prior autoimmune diseases. Individuals with a family history of an inflammatory rheumatic disease were excluded. 62 of the normal controls were Caucasian and 1 was Asian Indian. 50 of the 63 controls were from the same population base as the local patients.

DNA analysis. Peripheral blood mononuclear cells were used as a source for genomic DNA. Allele-specific oligonucleotide primers specific for the sequence polymorphism within the first hypervariable region (HVR) and for a nonpolymorphic stretch from amino acid positions 87-94 were used to amplify HLA-DRB1 genes. A polymerase chain reaction with 30 cycles was used under the following conditions: denaturation for $2 \mathrm{~min}$ at $94^{\circ} \mathrm{C}$, annealing for $2 \mathrm{~min}$ at $55^{\circ} \mathrm{C}$, and extension for $2 \mathrm{~min}$ at $72^{\circ} \mathrm{C}$. To identify sequence polymorphisms encoded by the second and third HVR of the HLA-DRB1 alleles, amplified products were blotted onto nylon membranes and hybridized with oligonucleotide probes specific for sequence polymorphisms of the different DRB1 alleles. The primer sets and the oligonucleotide probes used for the identification of the HLA-DRB1 alleles have been recently described $(16,17)$. Sequence polymorphism at position 57 within the HLA-DRB ${ }^{*} 04$ alleles, which is infrequent in a Caucasian population, was not determined. Thus, the approach did not allow a distinction between DRB ${ }^{*} 0404$ and ${ }^{*} 0405$. For hybridization, oligonucleotide probes were labeled with digoxygenin UTP and subsequently developed with an antidigoxygenin alkaline phosphatase-labeled antibody.

Statistical analysis. Demographic and clinical characteristics of the patient subsets and the frequencies of HLA-DRB1 alleles in patients and normals were compared by $t$ test, Chi-square test, or a Fisher's exact probability test as appropriate. Accumulated incidence rates were calculated using the equation $1-e-\sum_{i=0}^{i=72} i$; where $i=$ yearly incidence
rates.

\section{Results}

Overrepresentation of two allelic variants of the $H L A$ $D R B 1{ }^{*} 04$ haplotype in GCA patients. 42 patients with biopsyproven GCA were enrolled into this study. The group included
9 males and 33 females. Allele-specific amplification of genomic DNA was used to identify both HLA-DRB1 alleles in each patient. As shown in Table I, the distribution of HLA-DRB1 alleles among GCA patients was different from that in normals. HLA-DRB $1{ }^{*} 04$ was present in $59.5 \%$ of the patients. Three allelic variants, B1*0401, B1 *0404, and B1*0408, were identified in the patient cohort. B1 *0404 and *0408 are distinct only at position $86(\mathrm{~V} \rightarrow \mathrm{G})$ and were grouped together. Three of seven patients typed HLA-DRB $1 * 0408$. Interestingly, none of the HLA-DRB $1{ }^{*} 04^{+}$patients used the B1 ${ }^{*} 0402$ and B1 *0403 allele. These two alleles are rare in the normal population. The second most frequent allele was HLA-DRB1*03. Overrepresentation of HLA-DRB $1 * 03$, however, did not reach statistical significance. The overexpression of HLA-DRB 1 *04 within the GCA cohort was accompanied by a reduction of HLADRB1*01. A reduced frequency was also seen for HLA$\mathrm{DRB} 1 * 11, \mathrm{~B} 1 * 07$, and $\mathrm{B} 1 * 15 / 16$. An equal representation within patients and normals was characteristic for B1*08 and $\mathrm{B} 1 * 13$. We subsequently analyzed the role of allelic combinations in the patient group. Fig. 1 summarizes the haplotype combinations at the B1 locus for the 25 HLA-DRB $1 * 04^{+}$patients. In Fig. 2, both HLA-DRB1 alleles of the 17 HLADRB $1{ }^{*} 04^{-}$patients are shown. The HLA-DRB $1 * 04^{-}$patient subset was characterized by a nonrandom distribution of alleles. Three HLA-DRB1 variants were overrepresented: HLA$\mathrm{DRB} 1 * 03, \mathrm{~B} 1 * 08$, and $\mathrm{B} 1 * 13$. HLA-DRB $1 * 03$ was found in 8 of 17 patients compared with 11 of $48 \mathrm{DRB} 1^{*} 04^{-}$controls $(P$ $=0.06), \mathrm{DRB} 1{ }^{*} 08$ was found in 6 of 17 patients compared with 6 of 48 controls $(P=0.04)$. Only four patients were negative for all three alleles. These four patients shared the HLADRB $1 * 15 / 16$ haplotype. HLA-DRB $1 * 01$ and $* 07$ were clearly underrepresented in HLA-DRB ${ }^{*} 04^{-}$patients ( 5.8 vs. $27 \%, P$ $=0.06$; and 5.8 vs. $29 \%, P=0.04$, respectively) (Fig. 2 ). The HLA-DRB1 *0301 allele was not only frequently seen in nonDR4 patients, but was also preferentially expressed in combina-

Table I. Frequencies of HLA-DRBI Alleles in GCA Patients and Normals

\begin{tabular}{lcccc}
\hline DRB1 allele & $\begin{array}{c}\text { GCA patients } \\
(n=42)\end{array}$ & $\begin{array}{c}\text { Normals } \\
(n=63)\end{array}$ & $P$ & $P$ corr \\
\hline & $\%$ & $\%$ & & \\
01 (DR1) & 11.9 & 28.6 & 0.043 & NS \\
$15 / 16$ (DR2) & 26.2 & 33.3 & NS & NS \\
03 (DR3) & 28.6 & 19.0 & NS & NS \\
04 (DR4) & 59.5 & 23.8 & 0.0002 & 0.003 \\
0401 & 42.9 & 15.9 & 0.002 & 0.03 \\
0402 & 0.0 & 1.6 & NS & NS \\
0403 & 0.0 & 4.8 & NS & NS \\
$0404 / 8$ & 16.7 & 3.2 & 0.016 & NS \\
11 (DR5) & 9.5 & 22.2 & NS & NS \\
07 (DR7) & 14.3 & 22.2 & NS & NS \\
08 (DRw8) & 14.3 & 11.1 & NS & NS \\
09 (DR9) & 0.0 & 3.2 & NS & NS \\
10 (DRw10) & 0.0 & 3.2 & NS & NS \\
13 (DRw13) & 21.4 & 19.0 & NS & NS \\
14 (DRw14) & 2.4 & 3.2 & NS & NS \\
\hline
\end{tabular}

* Corrected for number of variables tested. 


\begin{tabular}{|c|c|c|c|c|c|}
\hline & & First $\mathrm{t}$ & 31 Allele & & $\begin{array}{l}\text { Second } \\
\text { DRB1 Allele }\end{array}$ \\
\hline & 401 & 402 & 403 & $404 / 8$ & \\
\hline GCA-1 & $\oplus \oplus$ & - & - & - & - \\
\hline GCA-2 & $\oplus$ & - & - & $\cdot$ & 01 \\
\hline GCA-3 & $\oplus$ & - & - & - & 01 \\
\hline GCA-4 & $\oplus$ & $\cdot$ & - & $\cdot$ & 01 \\
\hline GCA-5 & $\oplus$ & - & - & $\cdot$ & $15 / 16$ \\
\hline GCA-6 & $\oplus$ & - & - & - & 03 \\
\hline GCA-7 & $\oplus$ & $\cdot$ & - & - & 03 \\
\hline GCA-8 & $\oplus$ & $\cdot$ & - & - & 03 \\
\hline GCA-9 & $\oplus$ & - & - & - & 03 \\
\hline GCA-10 & $\oplus$ & - & - & - & 03 \\
\hline GCA-11 & $\oplus$ & $\cdot$ & - & - & 11 \\
\hline GCA-12 & $\oplus$ & - & - & - & 07 \\
\hline GCA-13 & $\oplus$ & - & - & - & 07 \\
\hline GCA-14 & $\oplus$ & - & - & - & 07 \\
\hline GCA-15 & $\oplus$ & - & - & $\cdot$ & 08 \\
\hline GCA-16 & $\oplus$ & - & $\cdot$ & $\cdot$ & 13 \\
\hline GCA-17 & $\oplus$ & $\cdot$ & $\cdot$ & $\cdot$ & 13 \\
\hline GCA-18 & $\oplus$ & $\cdot$ & - & - & 13 \\
\hline GCA-19 & $\cdot$ & $\cdot$ & - & $\oplus$ & 01 \\
\hline GCA-20 & - & $\cdot$ & - & $\oplus$ & $15 / 16$ \\
\hline GCA-21 & - & $\cdot$ & - & $\oplus$ & $15 / 16$ \\
\hline GCA-22 & - & - & - & $\oplus$ & $15 / 16$ \\
\hline GCA-23 & - & - & - & $\oplus$ & 03 \\
\hline GCA-24 & - & - & - & $\oplus$ & 07 \\
\hline GCA-25 & - & - & - & $\oplus$ & 07 \\
\hline
\end{tabular}

Figure 1. Allelic combinations at the HLA-DRB1 locus in HLADRB 1 *04-positive patients.

tions with one of the HLA-DRB $1 * 04$ variants (Fig. 1). Although no single HLA-DRB1 allelic variant can be pinpointed as a primary genetic component in GCA, the nonrandomness of HLA-DRB1 genes present in GCA patients suggests that a genetic element linked to the HLA class II complex is relevant in the pathogenesis of the disease.

Clinical presentation in $H L A-D R B 1^{*} 04^{+}$and $H L A$ $D R B 1{ }^{*} 04^{-}$GCA patients. Genotyping for the HLA-DRB1 locus indicated that individuals expressing one of two allelic variants, B1*0401 and B1 ${ }^{*} 0404 / 8$, had a higher risk to develop GCA. However, $40.5 \%$ of all patients were negative for the two disease-associated haplotypes. We therefore addressed the question whether the clinical presentation of the vasculitic syndrome was different in HLA-DRB ${ }^{*} 04^{+}$and HLA$\mathrm{DRB} 1{ }^{*} 04^{-}$patients. The patient group was analyzed for the following parameters: age at disease onset, sex, symptoms of PMR, relapses of symptoms in patients who were diagnosed before 1989, and permanent visual impairment. As demonstrated in Table II, expression of the HLA-DRB $1 * 04$ alleles did not affect the age at disease onset and symptoms of PMR were found in similar frequencies in both patient subsets. In general, all patients responded well to the initial high-dose corticosteroid therapy. Both patient subsets included individuals who relapsed and required the resumption of steroid treatment. Among the 17 HLA-DRB ${ }^{*} 04^{-}$patients, 3 received methotrexate or azathioprine in addition to prednisone. None of the HLA-DRB $1{ }^{*} 04^{+}$patients was treated with an additional immunosuppressive agent. Interestingly, we identified four pa-

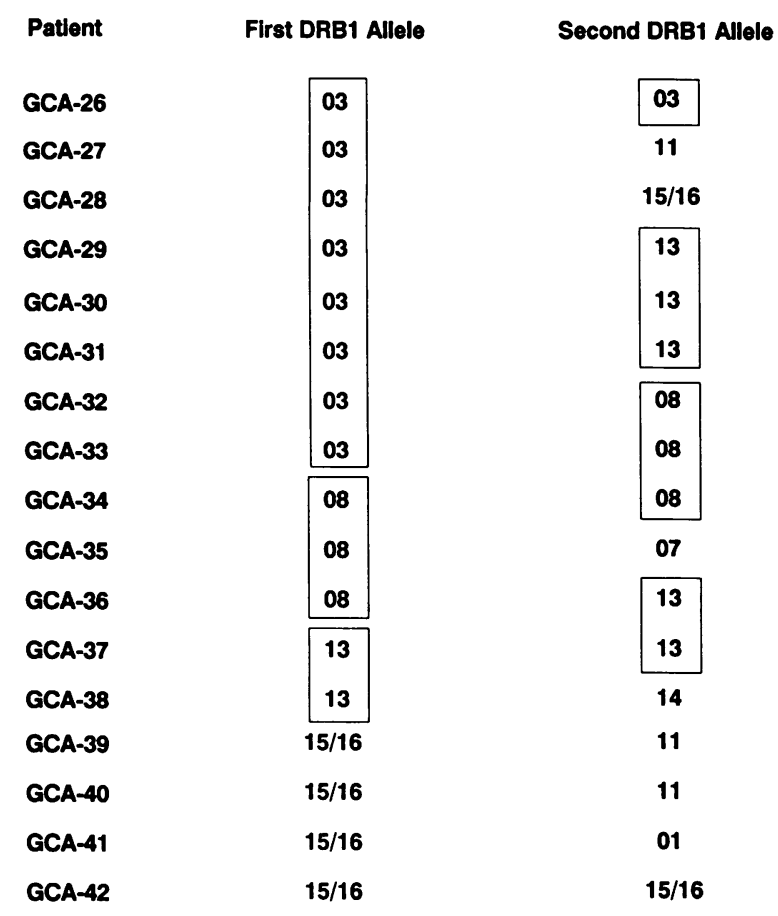

Figure 2. Allelic combinations at the HLA-DRB1 locus in HLADRB 1 *04-negative patients.

tients with permanent vision loss due to ischemic opticus neuritis. All four patients carried the B $1^{*} 0401$ or $B 1 * 0404 / 8$ allele. This finding indicates that the HLA-DRB $1 * 04^{+}$patients might develop more extended disease with more rapid progression.

Two HLA-DRBI *04-associated diseases, GCA and RA, are not linked. The allelic variants of HLA-DRB $1{ }^{*} 04$ that are overrepresented in GCA patients are the risk-conferring alleles for yet another autoimmune disease, RA. The seropositive form of RA is tightly linked to a sequence motif encoded by the HLA-DRB1 locus, which has been mapped to the HVR 3 of the HLA-DRB1 locus. The RA-associated sequence motif is most frequently found integrated into the HLA-DRB $1 * 04$ haplotype, but it can also be integrated into the HLA-DRB $1 * 0101$ and *1402 alleles. The common association of GCA and RA with the allelic subtypes DRB $1{ }^{*} 0401$ and ${ }^{*} 0404 / 8$ raises the question whether there is an increased risk for HLADRB $1{ }^{*} 04^{+}$individuals to develop both diseases. Both syndromes are more common in females, but the age of disease onset may be decades apart. To investigate whether both HLA-

Table II. Clinical Characteristics of GCA Patients

\begin{tabular}{|c|c|c|}
\hline & $\begin{array}{c}\text { HLA-DRB } 1^{*} 04^{+} \\
\text {patients } \\
(n=25)\end{array}$ & $\begin{array}{c}\text { HLA-DRB } 1 * 04- \\
\text { patients } \\
(n=17)\end{array}$ \\
\hline Age at disease onset (yr) & $71.2 \pm 8.4$ & $72.9 \pm 7.9$ \\
\hline Sex (\% females) & 76.0 & 82.4 \\
\hline $\begin{array}{l}\text { Symptoms of polymyalgia } \\
\text { rheumatica }(\%)\end{array}$ & 56.0 & 64.7 \\
\hline Permanent visual impairment (\%) & 16.7 & 0.0 \\
\hline $\begin{array}{l}\text { Number of patients requiring } \\
\text { prednisone therapy for }>2 \mathrm{yr}\end{array}$ & $7 / 9$ & $4 / 5$ \\
\hline
\end{tabular}


DRB 1 *04-linked diseases are found to frequently co-occur, we analyzed a cohort of 835 consecutive patients with tissue-confirmed diagnosis of GCA. This cohort included all patients with GCA from 1976 to 1990 who had a positive temporal artery biopsy within this institution. Among the 835 patients, 22 were diagnosed to suffer from polyarthritis before, concomitant with, or after the onset of GCA. On review of the records of these 22 patients, we found that 1 patient was falsely entered as GCA. The 21 patients were analyzed to identify the underlying condition leading to the diagnosis of polyarthritis. The results of the analysis are given in Table III. We only found one patient who had seropositive RA and was diagnosed to have GCA. He had developed RA in 1937 and received salicylates, corticosteroids, and finally parental gold. The disease took a destructive and nodular course. Over the several years before the diagnosis of GCA his joint disease was stable and not explicitly active. In 1976, he was admitted with bitemporal headaches, malaise, and weight loss. Temporal artery biopsy showed granulomatous arteritis. Within the cohort of 835 patients, there were no female patients with a history of seropositive RA. The majority of patients in the cohort with the cooccurrence of GCA and polyarthritis had a seronegative variant of a symmetric polyarthritis, which in some cases presented with joint space narrowing and erosions. This type of seronegative polyarthritis has been described as a manifestation of joint disease in GCA patients. The accumulated incidence rate for RA in a population with an age of $72 \mathrm{yr}$ was 19 of 606 females and 3.4 of 229 males (15). These data indicate that the risk of an HLA-DRB $1 * 04^{+}$ individual to develop seropositive RA and GCA is very low.

$A$ sequence motif shared by $H L A-D R B 1^{*} 04^{+}$and $H L A-$ $D R B 1{ }^{*} 04^{-} G C A$ patients. RA has been linked to a set of HLADRB1 genes, all of which share the disease-associated sequence motif in the HVR 3 of the HLA-DR $\beta 1$ chain. The most important members of the group of alleles conferring the risk to develop RA are the HLA-DRB $1 * 0401$, HLA-DRB1 *0404/8, and HLA-DRB 1 *0101 alleles. Data presented in Table I demonstrate that the HLA-DRB $1{ }^{*} 0101$ allele is not enriched in GCA patients. This allele is rather diminished, in particular, in the HLA-DRB ${ }^{*} 04^{-}$GCA population (Fig. 2), indicating that the sequence polymorphism of the HVR3 found in RA is not relevant for the risk of GCA patients to develop the vasculitis. The genotyping for the HLA-DRB1 genes in GCA revealed an overrepresentation of HLA-DRB ${ }^{*} 04$ alleles but also a nonrandom spectrum of non-DRB $1 * 04$ haplotypes. This finding raised the possibility that a region of sequence homology integrated into HLA-DRB $1 * 04$, but also into some of the $\mathrm{DRB} 1{ }^{*} 04^{-}$gene products, was shared by GCA patients. To identify a common denominator among the GCA patients, we compared the sequence polymorphism of the HLA-DRB1 alleles $\mathrm{B} 1 * 0301, \mathrm{~B} 1 * 1301 / 2$, and $\mathrm{B} 1 * 0801$ frequently found in DRB $1 * 04-$ GCA patients with B1*0401 and *0404/8. The disease-associated alleles share a sequence motif encoded by the HVR2; in particular, the polymorphic amino acid positions 28, 30, and 31 are identical (Fig. 3). The amino acid sequence motif DRYF, which was expressed by all 42 GCA patients, is not present in the HLA-DRB1 alleles *01 and *07, which were found to be decreased in the GCA population. The recent resolution of the three-dimensional structure of an HLA molecule has allowed correlation of polymorphic sites with functional regions of the molecule (18). Human HLA-DR molecules are expressed on the cell surface as a heterodimer. $\beta$ pleated sheets encoded by the HVR 1 and HVR 2 of the $\alpha$ and $\beta$ chain form the bottom of a groove that is surrounded by two $\alpha$-helical formations building the walls of the cleft (Fig. 4). There is overwhelming evidence that the groove represents the binding site for antigenic peptides that are embedded between the $\alpha$-helical walls. The complex formed by the antigenic peptide bound in the groove of the HLA molecules represents the ligand for the $\mathrm{T}$ cell receptor and is required to initiate $\mathrm{T}$ cell activation. Thus, our data indicate that a polymorphic region

Table III. Co-occurrence of GCA and RA

\begin{tabular}{|c|c|c|c|c|c|}
\hline & \multirow[b]{2}{*}{ Total } & \multicolumn{2}{|c|}{ Found } & \multicolumn{2}{|c|}{ Expected } \\
\hline & & Female & Male & Female & Male \\
\hline & \multicolumn{5}{|c|}{$n$} \\
\hline Biopsy-proven GCA & 835 & 606 & 229 & & \\
\hline Patients with polyarthritis & 22 & 19 & 3 & & \\
\hline Misentry & 1 & 1 & 0 & & \\
\hline Patients with GCA and polyarthritis & 21 & 18 & 3 & & \\
\hline \multicolumn{6}{|l|}{ Disease entity causing polyarthritis } \\
\hline Polyarthritis in GCA & 11 & 9 & 2 & & \\
\hline Osteoarthritis & 3 & 3 & 0 & & \\
\hline Gout & 1 & 1 & 0 & & \\
\hline Seronegative monoarthritis/oligoarthritis & 2 & 2 & 0 & & \\
\hline Systemic lupus erythematosus & 1 & 1 & 0 & & \\
\hline Rheumatoid arthritis & 3 & 2 & 1 & $19 / 606$ & $3.4 / 229$ \\
\hline Seronegative, nonerosive & 2 & 2 & 0 & & \\
\hline Seropositive & 1 & 0 & 1 & & \\
\hline
\end{tabular}

The Mayo Clinic data bank was used to estimate how frequently patients with biopsy-proven GCA carried the diagnosis of RA. 835 patients with GCA were registered between 1976 and 1990, 22 were diagnosed to have polyarthritis. Charts of these 22 patients were reviewed. Expected prevalence rates in 72-yr-old patients were calculated as the accumulated incidence rates for a cohort of 606 female and 229 male individuals from the data published by Linos et al. (15). 
HVR1 HVR3

\begin{tabular}{|c|c|c|c|c|c|c|c|c|c|c|c|c|c|c|c|c|c|c|c|c|c|}
\hline AA position & 49101 & 11 & 12 & & 14 & 26 & 28 & 30 & 31 & 323 & 33 & 37 & 57 & 58 & 60 & 6770 & 71 & 73 & 74 & 78 & 86 \\
\hline \multicolumn{22}{|l|}{ DRB1 allele } \\
\hline \multicolumn{22}{|c|}{ Disease-associated } \\
\hline 401 & R E $\mathbf{Q}$ & $\mathbf{v}$ & $\mathbf{K}$ & H & $\mathbf{E}$ & $\mathbf{F}$ & D & $\mathbf{Y}$ & $\mathbf{F}$ & $\mathbf{Y}$ & H & $\mathbf{Y}$ & D & $\mathbf{A}$ & $\mathbf{Y}$ & $\mathbf{L} \mathbf{Q}$ & $\mathbf{K}$ & A & $\mathbf{A}$ & $\mathbf{Y}$ & G \\
\hline $404 / 8$ & R E Q & v & $\mathbf{K}$ & H & $\mathbf{E}$ & $\mathbf{F}$ & D & $\mathbf{Y}$ & $\mathbf{F}$ & $\mathbf{Y}$ & H & $\mathbf{Y}$ & D & A & $\mathbf{Y}$ & $\mathbf{L} \mathbf{Q}$ & $\mathbf{R}$ & A & A & $\mathbf{Y}$ & $\mathbf{v}$ \\
\hline 0301 & R E Y & $\mathbf{s}$ & $\mathbf{T}$ & $\mathbf{s}$ & $\mathbf{E}$ & $\mathbf{Y}$ & D & $\mathbf{Y}$ & $\mathbf{F}$ & H & $\mathbf{N}$ & $\mathbf{N}$ & D & A & $\mathbf{Y}$ & $\mathbf{L} \mathbf{Q}$ & $\mathbf{K}$ & G & $\mathbf{R}$ & $\mathbf{Y}$ & v \\
\hline 1301 & R E Y & $\mathbf{s}$ & $\mathbf{T}$ & $\mathbf{s}$ & $\mathbf{E}$ & $\mathbf{F}$ & D & $\mathbf{Y}$ & $\mathbf{F}$ & H & $\mathbf{N}$ & $\mathbf{N}$ & D & A & $\mathbf{Y}$ & I D & $\mathbf{E}$ & A & A & $\mathbf{Y}$ & v \\
\hline 0801 & R E Y & $\mathbf{s}$ & $\mathbf{T}$ & $\mathbf{R}$ & $\mathbf{E}$ & $\mathbf{F}$ & D & $\mathbf{Y}$ & $\mathbf{F}$ & $Y$ & $\mathbf{N}$ & $\mathbf{Y}$ & $\mathbf{s}$ & A & $\mathbf{Y}$ & F D & $\mathbf{R}$ & A & $\mathbf{L}$ & $\mathbf{Y}$ & G \\
\hline 1501 & R $\mathbf{W} \mathbf{Q}$ & $\mathbf{P}$ & $\mathbf{K}$ & $\mathbf{R}$ & $\mathbf{E}$ & $\mathbf{F}$ & D & $\mathbf{Y}$ & $\mathbf{F}$ & $\mathbf{Y}$ & H & $\mathbf{s}$ & D & $\mathbf{A}$ & $\mathbf{Y}$ & 10 & A & A & A & $\mathbf{Y}$ & $\mathbf{v}$ \\
\hline \multicolumn{22}{|c|}{ Disease non-associated } \\
\hline 0101 & R $\mathbf{w} \mathbf{Q}$ & $\mathbf{L}$ & $\mathbf{K}$ & $\mathbf{F}$ & $\mathbf{E}$ & $\mathbf{L}$ & $\mathbf{E}$ & c & $\mathbf{I}$ & $\mathbf{Y}$ & $\mathbf{N}$ & $\mathbf{s}$ & D & A & $\mathbf{Y}$ & $\mathbf{L} \mathbf{Q}$ & $\mathbf{R}$ & $\mathbf{A}$ & $\mathbf{A}$ & $\mathbf{Y}$ & $\mathbf{G}$ \\
\hline 0701 & $\mathbf{a} \mathbf{w}$ & G & $\mathbf{K}$ & $\mathbf{Y}$ & $\mathbf{K}$ & $\mathbf{F}$ & $\mathbf{E}$ & $\mathbf{L}$ & $\mathbf{F}$ & $\mathbf{Y}$ & $\mathbf{N}$ & $F$ & v & A & $\mathbf{s}$ & I D & R & G & $\mathbf{Q}$ & $\mathbf{v}$ & G \\
\hline
\end{tabular}

Figure 3. Conserved region of sequence polymorphism in HLA-DRB1 alleles expressed by GCA patients.

on the floor of the antigen binding site of the HLA-DR molecule is shared by patients who develop GCA (Fig. 4).

\section{Discussion}

A lymphocytic infiltrate in the walls of medium- and largesized arteries is the characteristic finding in GCA $(4,5)$. Important clinical features associated with the vasculitis are the frequent acute onset of symptoms, a brisk production of acute phase reactants, and the prompt response of symptoms and acute phase proteins to corticosteroids. Evidence for a crucial role of $\mathrm{T}$ lymphocytes comes from the demonstration of $\mathrm{CD}^{+}$ $T$ helper cells constituting the dominating cell population in the inflammatory infiltrate and reports describing a selective depletion and activation of $\mathrm{CD}^{+} \mathrm{T}$ cells in the peripheral blood $(5,19)$. The goal of this study was to investigate HLA molecules that exert a considerable control of $T$ cell function. $\mathrm{CD}^{+} \mathrm{T}$ cells recognize foreign antigen only when displayed in association with self-HLA-DR molecules (6). Thus, HLA molecules are an absolute requirement for selection and binding of antigenic determinants and the subsequent triggering of the $T$ cell receptor.

The polymorphism of HLA-DR molecules is mainly determined by the highly polymorphic HLA-DRB1 locus for which an estimated 80 alleles have been described (20). Here, we report that the expression of HLA-DRB1 alleles by patients with GCA is nonrandom. $60 \%$ of the patients carried one of the closely related allelic variants, HLA-DRB1 *0401 and *0404/ 8. The difference between these three alleles is limited to a polymorphism in residue positions 71 and 86 of the $B 1$ gene product. Two other allelic products of the HLA-DRB 1 *04 family, B ${ }^{*} 0402$ and $* 0403$, were not identified within the patient population.

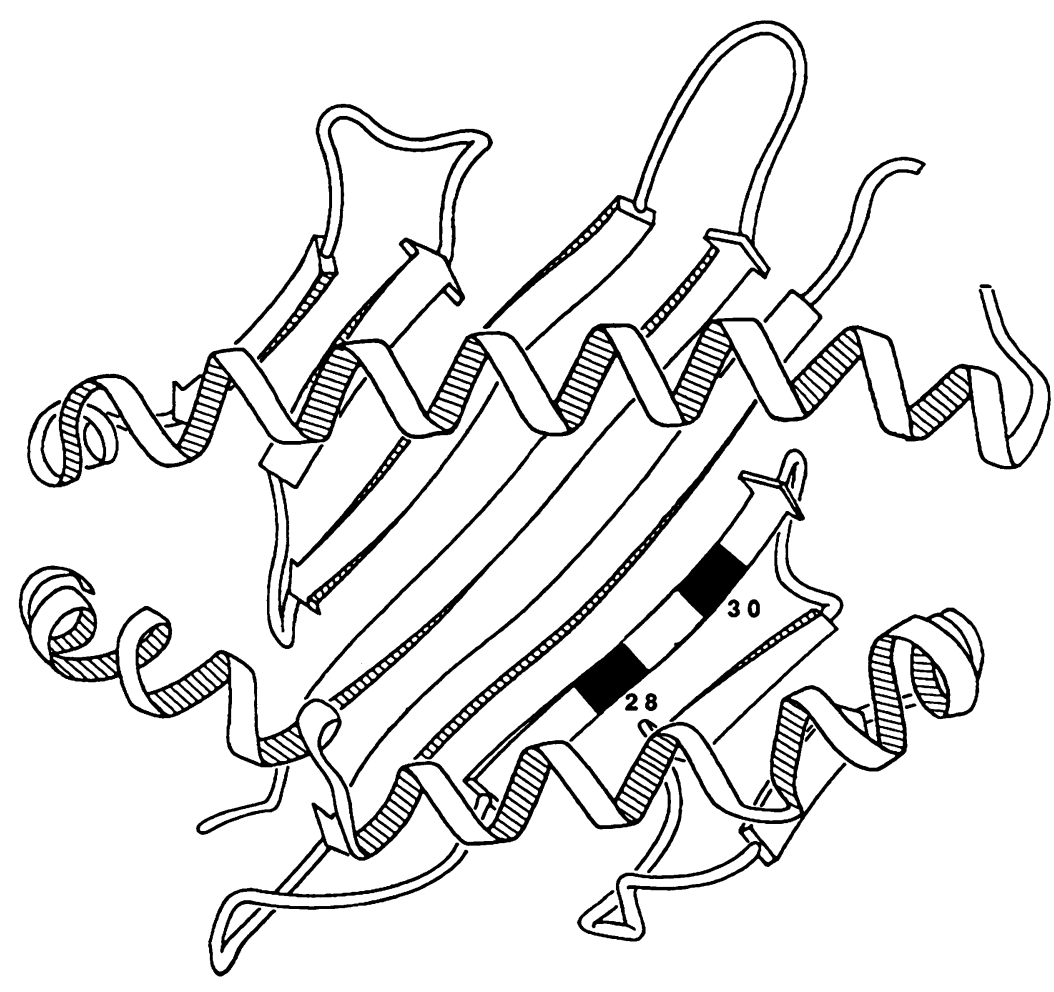

Figure 4. Mapping of the GCA-linked sequence motif to the antigen-binding site of the HLA-DR molecule. The HLA-DR sequence was modeled on the crystal structure of the HLA-A2 as described by Brown et al. (18). The conserved tetrapeptide DRYF encompassing amino acid positions 28-31 maps to the antigen-binding groove. Amino acid positions 28 and 30 are predicted to point up and are highlighted as solid black squares. 
Although an HLA-DR association of the disease has been reported, data have been controversial. Several studies have attempted to identify a GCA-associated haplotype (7-11). All available data used conventional techniques and could not distinguish among allelic variants of the DRB $1{ }^{*} 04$ family. In all reports, the frequency of HLA-DR4 was increased, although the difference among the patient population and normal controls did not always reach statistical significance. Interestingly, the HLA-DR4 association of GCA is supported by differences in the incidence of the disease in distinct ethnic groups. An increased frequency of the vasculitis in Caucasians compared with black individuals has been described. The highest incidence has been reported in Scandinavia and Minnesota, mainly including a population of Northern European descent $(2,3)$. Studies of Italian and Israeli populations have demonstrated low frequencies $(21,22)$. These ethnic differences correlate to the frequency of HLA-DR4 expressed in the different populations.

The finding that $40 \%$ of the patients were negative for B $1 * 0401$ and B $1 * 0404 / 8$ indicated that the contribution of the HLA-DR molecule in GCA is probably not related to a unique function of the DRB $1{ }^{*} 04$ allele. We hypothesized that the disease is linked to a sequence polymorphism present in the two frequent HLA-DRB 1 *04 alleles but also expressed in nonDRB $1 * 04$ alleles. This hypothesis was supported by our finding that the distribution of the HLA-DRB1 genes in HLADRB $1{ }^{*} 04^{-}$patients was nonrandom. HLA-DRB $1 * 03, \mathrm{~B} 1 * 13$, and $\mathrm{B} 1{ }^{*} 08$ were more frequent in the HLA-DRB ${ }^{*} 04^{-}$patient population than in the control population. Sequence comparison demonstrated that all alleles overrepresented in the patient population had a discrete region of sequence identity stretching from residues 28 to 31 . This sequence stretch includes one nonpolymorphic residue in position 29 . The sequence motif DRYF was found in all 42 GCA patients. Interestingly, two haplotypes frequently encountered in the normal population, HLA-DR 1 and HLA-DR7, carry very different amino acid residues at positions 28,30 , and 31 . The HLA-DR 1 haplotype was underrepresented in the GCA population. Expression of HLADR7 was limited to GCA patients who expressed the diseaselinked sequence cassette DRYF on their second haplotype. These data suggested that a single copy of the disease-associated sequence stretch is sufficient to confer disease susceptibility and that sequence variations in residues 28,30 , and 31 on the second haplotype appear not to be protective. The sequence stretch DRYF is also found in the DRB1*11 and DRB $1 * 15 / 16$ alleles, and in the 0101 and 0301 alleles of the HLA-DRB3 locus. HLA-DRB1*11 was infrequent in the total GCA population; HLA-DRB $1 * 15 / 16$ was found in the four patients who typed negative for HLA-DRB1 alleles $03,04,08$, and 13. The finding that the presence of the motif DRYF by itself does not necessarily confer susceptibility to GCA indicates that other regions of the HLA-DR $\beta 1$ chain have a modulatory effect on the biological function of the HLA-DR molecule, resulting in an increased risk to develop GCA. This interpretation is supported by the finding that there is a clear hierarchy of HLA-DRB1 alleles predisposing for GCA, with HLA-DRB $1 * 0401$ being the strongest risk factor. The existence of distinct roles for different disease-associated alleles sharing a particular sequence stretch is not unique for GCA but is also observed for RA. The HLA-DRB1 alleles *0101, *0401, *0404/8, and *1402, which are associated with RA and share a sequence stretch in the HVR3, clearly have distinct impacts in susceptibility and severity of the disease (17).

It could be argued that the HLA-DRB 1 locus does not represent the primary genetic factor linked to GCA. HLA haplotypes include multiple loci that are characterized by a linkage disequilibrium. No HLA-DQA or B allele can be identified that could represent a common denominator among the DR4, DR3, DRw8, and DR 13 haplotypes. In line with this finding, previous studies have failed to establish an HLA-DQ association of the disease (8).

The comparison of the clinical findings in the DR4 ${ }^{+}$and DR4- patient subsets also supported the view that the patients suffered from a similar disease entity. Especially, we could not confirm the results of a study applying metaanalysis to evaluate the role of HLA-DR4 in GCA (10). These authors discussed that the overrepresentation of HLA-DR4 might be caused by a linkage of HLA-DR4 to PMR. In our clinically well-characterized patient cohort, symptoms of PMR were encountered as frequently in the DR4- $4^{-}$as the DR4 ${ }^{+}$patient subset. Interestingly, all patients who had developed blindness due to ischemic opticus neuritis carried the HLA-DRB1 ${ }^{*} 0401$ or ${ }^{*} 0404 / 8$ allele. In early studies of GCA, blindness was a much more frequent complication. The increased awareness of physicians probably has lead to early diagnosis and treatment before irreversible ischemic damage has evolved. However, the finding of a higher frequency of blindness in the HLA-DRB $1{ }^{*} 0401 / 4$ / $8^{+}$group may indicate that the disease progresses at a faster rate in these individuals, suggesting a potential role of these allelic variants in disease severity.

Extensive studies on yet another functional region of the HLA-DRB $1{ }^{*} 04$ alleles have demonstrated that a discrete portion of the HLA-DR molecule can function as the primary genetic unit underlying HLA and disease association. A set of alleles at the DRB1 locus, including HLA-DRB $1 * 0401$, ${ }^{*} 0404 / 8,{ }^{*} 0101$, and ${ }^{*} 1402$, have been directly implicated in the pathogenesis of seropositive RA (23-27). The polymorphism that is shared by this set of RA-associated alleles but distinct from several closely related HLA class II alleles that are not associated with RA has been mapped to a short sequence stretch of DRB1 alleles spanning codons 67-74 (28-30). Here, we present data that a different segment of the DRB1 gene found in B1 ${ }^{*} 0401$ and B1 ${ }^{*} 0404 / 8$ accounts for the association of DRB $1 * 04$ alleles with GCA. This interpretation is supported by the underrepresentation of HLA-DR 1 in the GCA population, indicating that the sequence polymorphism within the HVR3 region of the gene is not relevant for the risk to develop GCA. Our finding that two different polymorphic domains of the HLA-DR molecule are linked to two separate disease entities suggests that distinct biological functions of the HLA-DR4 molecules are important in the pathogenesis of GCA and RA. This hypothesis is strengthened by epidemiologic data analyzing the cooccurrence of RA and GCA. In the consecutive case series of 835 patients with biopsy-proven GCA, we identified only 1 patient who had classical seropositive RA, a male who developed arthritis before GCA. There were no female patients with seropositive RA. This indicates that the cooccurrence of these two syndromes is certainly not increased, even though they share a genetic marker. The results of the case series are consistent with the paucity of reports in the literature describing GCA in patients who previously or subsequently developed RA (31). Such cases have been reported from this institution 
and include the one case identified in our current case series analysis. Some reports have indicated an increased mortality of RA patients, which, however, is not sufficient to explain the low cooccurrence of both diseases. There is no reason to believe that cases with both GCA and RA would not be recognized clinically. Rather, these data suggest the possibility that GCA patients may have an actually reduced risk to develop RA. The exact pathological mechanisms leading to seropositive RA are not understood, although a crucial role of T lymphocytes in the disease has been established. It could be speculated that the immune abnormalities characteristic for RA prevent an abnormal immune response resulting in granulomatous vasculitic vessel wall infiltrates in GCA.

The definition of a sequence cassette linked with disease susceptibility is important for our understanding of immune functions in GCA patients. Experimental evidence has been provided demonstrating that a discrete cluster of polymorphic sites within an HLA-DR sequence can function as a functional unit on different allelic backgrounds. Defining the sequence stretch DRYF as the genetic element predisposing to GCA raises the question on the importance of this unit in immunological recognition. Modeling of the HLA-DR molecule predicts that a discrete antigen binding groove is surrounded on both sides by $\alpha$-helical loops (18). In this model, residues 5686 of the $\beta$ chain, including the shared epitope linked to RA, form one of the $\alpha$-helical loops. In contrast, residues 28-31 of the $\beta$ chain linked to GCA would contribute to the floor of the peptide binding cleft. Data derived from detailed studies of the functional consequences of sequence variations in murine MHC molecules indicate that the amino acid in position 28 is of crucial importance for the selective binding of antigen fragments (18). Our data suggest that further analysis of the role of this residue in the immune response of GCA may provide information on the possibility that a specific disease-causative antigen might be bound by the disease-linked HLA polymorphism that initiates the vasculitic reaction in the arterial wall.

\section{Acknowledgments}

We thank Miss T. L. Buss for providing us with secretarial assistance.

This work was supported in part by a grant from the American Heart Association and the Mayo Foundation.

\section{References}

1. Calamia, K. T., and G. G. Hunder. 1980. Clinical manifestations of giant cell (temporal arteritis). Clin. Rheum. Dis. 6:389-403.

2. Huston, K. A., G. G. Hunder, J. T. Lie, R. H. Kennedy, and L. R. Elveback. 1978. Temporal arteritis: a 25-year epidemiologic, clinical, and pathologic study. Ann. Intern. Med. 88:162-167.

3. Machado, E. B. V., C. J. Michet, D. J. Ballard, G. G. Hunder, C. M. Beard, C.-P. Chu, and W. M. O'Fallon. 1988. Trends in incidence and clinical presentation of temporal arteritis in Olmsted County, Minnesota, 1950-1985. Arthritis Rheum. 31:745-749.

4. Lie, J. T., and Members and Consultants of the American College of Rheumatology. Subcommittee on Classification of Vasculitis. 1990. Illustrated histopathologic classification criteria for selected vasculitis syndromes. Arthritis Rheum. 33:1074-1087.

5. Banks, P. M., M. D. Cohen, W. W. Ginsburg, and G. G. Hunder. 1983. Immunohistologic and cytochemical studies of temporal arteritis. Arthritis Rheum. 26:1201-1207.

6. Schwartz, R. H. 1986. Immune response (Ir) genes of the murine major histocompatibility complex. Adv. Immunol. 38:31-201.

7. Calamia, K. T., S. B. Moore, L. R. Elveback, and G. G. Hunder. 1981.
HLA-DR locus antigens in polymyalgia rheumatica and giant cell arteritis. $J$ Rheumatol. 8:993-996.

8. Bignon, J. D., C. Ferec, J. Barrier, Y. Pennec, C. Verlingue, M. L. Cheneau, V. Lucas, J. Y. Muller, and J. P. Saleun. 1988. HLA class II genes polymorphism in DR4 giant cell arteritis patients. Tissue Antigens. 32:254-258.

9. Lowenstein, M. B., P. H. Bridgeford, F. B. Vasey, B. F. Germain, and L. R. Espinoza. 1983. Increased frequency of HLA-DR3 and DR4 in polymyalgia rheumatica-giant cell arteritis. Arthritis Rheum. 26:925-927.

10. Richardson, J. E., D. D. Gladman, A. Fam, and E. C. Keystone. 1987. HLA-DR4 in giant cell arteritis: association with polymyalgia rheumatica syndrome. Arthritis Rheum. 30:1293-1297.

11. Salvarani, C., P. Macchioni, F. Zizza, W. Mantovani, F. Rossi, C. Castri, N. Capozzoli, R. Baricchi, L. Boiardi, F. Chiaravalloti, et al. 1991. Epidemiologic and immunogenetic aspects of polymyalgia rheumatica and giant cell arteritis in Northern Italy. Arthritis Rheum. 34:351-356.

12. Ginsburg, W. W., M. D. Cohen, S. B. Hall, R. S. Vollertsen, and G. G. Hunder. 1985. Seronegative polyarthritis in giant cell arteritis. Arthritis Rheum 28:1362-1366.

13. Arnett, F. C., S. M. Edworthy, D. A. Bloch, D. J. McShane, J. F. Fries, N. S. Cooper, L. A. Healey, S. R. Kaplan, M. H. Liang, H. S. Luthra, et al. 1988 The American Rheumatism Association 1987 revised criteria for the classification of rheumatoid arthritis. Arthritis Rheum. 31:315-324.

14. Tan, E. M., A. S. Cohen, J. F. Freeze, K. T. Mayse, D. J. McChain, N. F. Rothfield, J. G. Shaller, N. Tallar, and R. J. Winchester. 1982. The 1982 revised criteria for the classification of systemic lupus erythematosus. Arthritis Rheum. 25:1271-1277

15. Linos, A., J. W. Worthington, W. M. O'Fallon, and L. T. Kurland. 1980. The epidemiology of rheumatoid arthritis in Rochester, Minnesota: a study of incidence, prevalence, and mortality. Am. J. Epidemiol. 111:87-98.

16. Weyand, C. M., K. C. Hicok, and J. J. Goronzy. 1991. Nonrandom selection of $T$ cell specificities in anti-HLA-DR responses: sequence motifs of the responder HLA-DR allele influence T cell recruitment. J. Immunol. 147:70-78.

17. Weyand, C. M., C. Xie, and J. J. Goronzy. 1992. Homozygosity for the HLA-DRB1 allele selects for extraarticular manifestations in rheumatoid arthritis. J. Clin. Invest. 89:2033-2039.

18. Brown, J. H., T. Jardetzky, M. A. Saper, B. Samraoui, P. J. Bjorkman, and D. C. Wiley. 1988. A hypothetical model of the foreign antigen binding site of class II histocompatibility molecules. Nature (Lond.). 332:845-850.

19. Dasgupta, B., O. Duke, A. M. Timms, C. Pitzalis, and G. S. Panayi. 1989. Selective depletion and activation of CD8 positive lymphocytes from peripheral blood in polymyalgia rheumatica and giant cell arteritis. Ann. Rheum. Dis. 48:307-311.

20. Marsh, S. G. E., and J. G. Bodmer. 1991. HLA class II nucleotide sequences. Immunogenetics. 33:321-334.

21. Friedman, G., B. Friedman, and J. Benbassat. 1982. Epidemiology of temporal arteritis in Israel. Isr. J. Med. Sci. 18:241-244.

22. Salvarani, C., P. L. Macchioni, P. L. Tartoni, F. Rossi, R. Baricchi, C. Castri, F. Chiaravalloti, and I. Portioli. 1987. Polymyalgia rheumatica and giant cell arteritis: a 5-year epidemiologic and clinical study in Reggio Emilia, Italy. Clin. Exp. Rheumatol. 5:204-215.

23. Stastny, P. 1978. Association of the B-cell alloantigen DRw4 with rheumatoid arthritis. $N$. Engl. J. Med. 298:869-871.

24. Nepom, G. T., S. Holbeck, C. E. Seyfried, K. P. Wilske, and B. S. Nepom 1986. Identification of HLA-Dw14 genes in DR4-positive RA. Lancet. ii:10021004.

25. Goronzy, J., C. M. Weyand, and C. G. Fathman. 1986. Shared T cell recognition sites on HLA class II molecules of patients with seropositive rheumatoid arthritis. J. Clin. Invest. 77:1042-1049.

26. Schiff, B., Y. Mizrachi, S. Orgad, M. Yaron, and I. Gazit. 1982. Association of HLA-Aw31 and HLA-DR 1 with adult rheumatoid arthritis. Ann. Rheum. Dis. 41:403-406.

27. Willkens, R. F., G. T. Nepom, C. R. Marks, J. W. Nettles, and B. S. Nepom. 1991. The association of HLA-Dw16 with rheumatoid arthritis in Yakima Indians: further evidence for the "shared epitope" hypothesis. Arthritis Rheum. 34:43-47.

28. Weyand, C. M., and J. J. Goronzy. 1989. Seropositive rheumatoid arthritis is associated with $\mathrm{T}$-cell epitopes encoded within the third hypervariable region of the HLA-DRI and the HLA-DR4 haplotype. In Immunobiology of HLA, Immunogenetics and Histocompatibility. Vol. II. B. Dupont, editor. Springer Verlag, New York 422-425.

29. Gregersen, P. K., J. Silver, and R. J. Winchester. 1987. The shared epitope hypothesis. Arthritis Rheum. 30:1205-1213.

30. Nepom, G. T., J. Hansen, and B. Nepom. 1987. The molecular basis for HLA class II associations with rheumatoid arthritis. J. Clin. Immunol. 7:1-7.

31. Hall, S., W. W. Ginsburg, R. S. Vollertsen, and G. G. Hunder. 1983. The coexistence of rheumatoid arthritis and giant cell arteritis. J. Rheumatol. 10:995997. 